\title{
Alman çoban köpeğinde articulatio temporomandibularis ve çiğneme kaslarının anatomisi*
}

\author{
Murat KABAK \\ Mustitiı Kemal Üniversitesi. Veteriner Fakiülesi. Anatomi Anabilim Dah. Hatlay
}

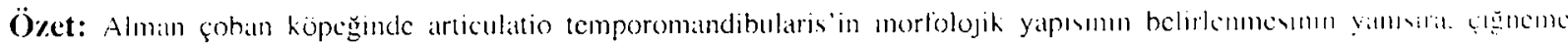

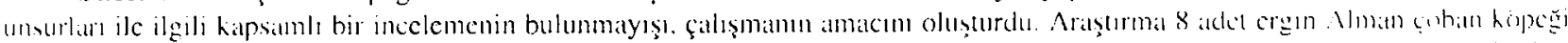

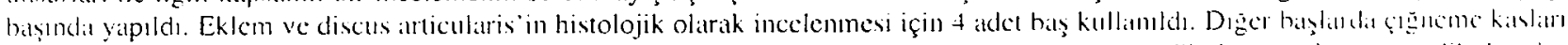

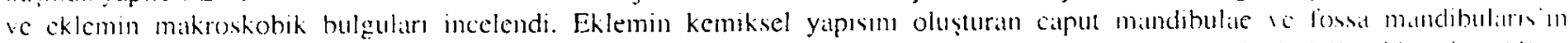

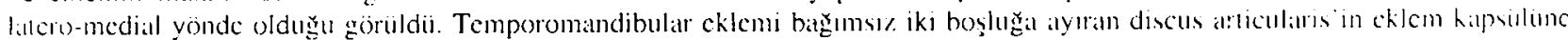

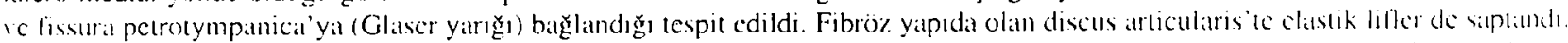

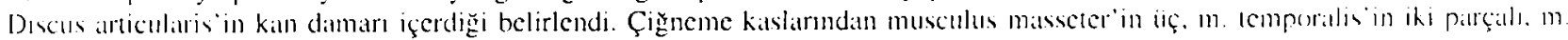

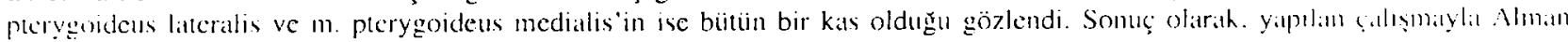

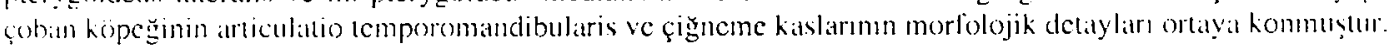

Anathtar kelimeler: Articulatio temporomandibularis. çăgneme kaslarr. discus articulatrs, mandibula
\end{abstract}

\section{The anatomy of the articulatio temporomandibularis and masticatory muscles of the (ierman shepherd dog}

Summary: The objective of the study was to establish the morpholngic structure of emporomandibulat femme in Germill shepherd dog due to the hact that there was no detailed research on the masticatory elements. In the study. S Germin vhepherd doges were used. Four heads were used to examine the joint and discus articularis histologically. The other heats were used fon

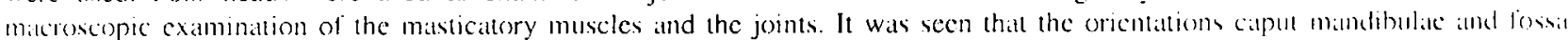
mindibularis. which form the bone structure of the joint were in latero-mediat direction. It wis cotablished that the juint divicied in

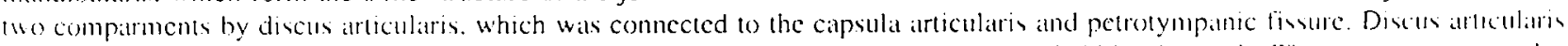

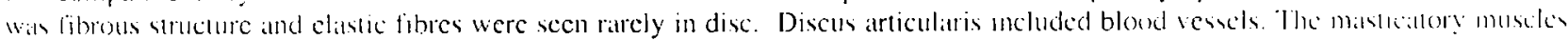

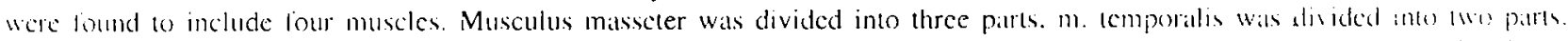

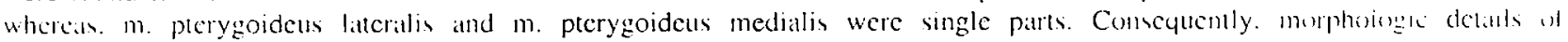
tomporomandibulial joint and masticatory muscles in the German shepherd dog were utilized by the preson study.

Key uords: Articulatio temporomandibularis. discus articularis. mandihula, masticatory muscler

\section{Giriş}

Çoğunlukla hayransal kalynaklarla beslenen Alman forhan kripeği, Carnivora takımmmn Canidea familyasmm bir ijesidir. Memelilerde farklı beslenme articulatio temporomandibularis'i şekillendiren unsurlarda. çiğneme kilslannda ve buna bağhl olarak mandibular hareketlerde larkliliklana neden olur $(4.8,12.13)$.

Ginglymoarthrodial oliarak smullandırlan bu cklem, mkongruent ginglymus olarak çalışı (15). Articulatio emporomandihularis’i oluşluran keniksel unsurlar arasmdaki uyumsuzluğun Camivora da en fazla olduğu bildirimişir (4.11,12.24). Eklem yiukteri arasmdaki bu uyumsu/luk. libroz ya da fibro-kartilaginoiz yapıda olan liecus aticularts ile ortadan kaldirihr. Bu discus, eklem buṣluğ unu (civum articulare) bağımsı iki boşluğa ayırır
$(9,10,12,17,18)$. Kikırdak dokudan meydana gelen discus articularis, malkroskobik (16.20.24) ve mikroskobik (12.13) olarak incelennuş̧ir. Temporomandihular cklemı $i$ saran eklem kapsulası (capsula articularis) ac bu kapsulii

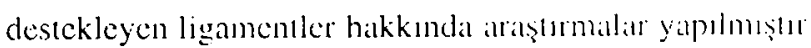
(1,24). Articulatio lemporomanclibularis de açullp-kapanma (rolasyon), ileri-geri (kayma) ve yamal (latcral) hareketler şeklinde ïç farklı çiğneme harekeli goiriblii (2,15). Köpeğin. hu harekellerin hepsini yapahilsc de. esas olarak w!asyon harcketini yaplığ bilchirilmişir (1?. 13.17.20.24).

Mandibula'mn harekellerini yapabilmesinde oncmlı bir elkiye sahip olan çiğneme kalsları hakkında falıs smilal yapımıs ve m. masseter, n. temporalis. m. pterygoicleus medialis ve m. perygoideus lateralis glarak incelenmisistir $(17.20 .24 .25)$.

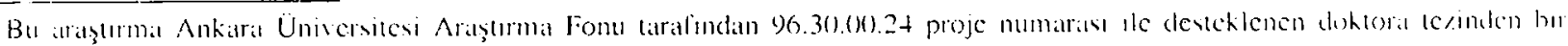
bolimom oreladir 
Bu çalışada. Almaın f̧oban köpeğinin articulatio lemporomandibularis ve çiğneme kaslarmm morfolojisi araşur yannula. Alman çoban köpeğinde bu denli kapsamlı bir

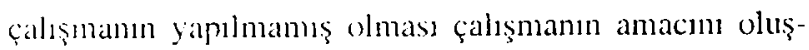
turmuştur.

\section{Materyal ve Metot}

Çalışmada. 8 adel erğin Alman çoban köpeği başı kullamsld. Hay anlarda kondrodistroli. hastallk ya da çenenin açılıp kapanmalsmnda bir sorun görülnedi. Hisonlojik incelemelerde kullanılacak başlarm (4 ades) perfiis:on işlemleri yapıldıktan sonra, diğer başlarla birlikte if 10 luk formol haruzuna konarak fikzasyonları sağlan (1).

Tüin bașlan median hallan kesilerek ikiye ayrild. D.)alha somra articulalio temporomandibularis ve çiğneme kisları makroskobik olarak incelendi. Çiğnenı kaslarmnn lil younleri kalà dïzlemine göre isimlendirildi. Lif seyirleri. discus articularis ve cklem stereomikroskop (Olympus MTX marki) ile incelendi. Histolojik incelenmeler jęin discus articularis\%ler laleral. central ve medial olmak iizere itę kısma ayrald. Eklem ise formik asit-Na sitrat (14) melodu ile dekalsifiye edildi. Discus articularis'ler(len horinomal. eklemden ise sagillal ve ransversal olarak alman onncklerden 5-6 mikron kalınlığnda histolojik preparatlar hayrlande. Discus articularis de bulunan kollagen denetler Weigert's clastik boyama yöntemi (14), claslik iplikler ise Orecin boyama yöntemi (7) ile beliflendi. Feklem ise. Crossmon (6)'un ieçlü boyama yön-

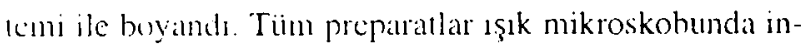
celendi.

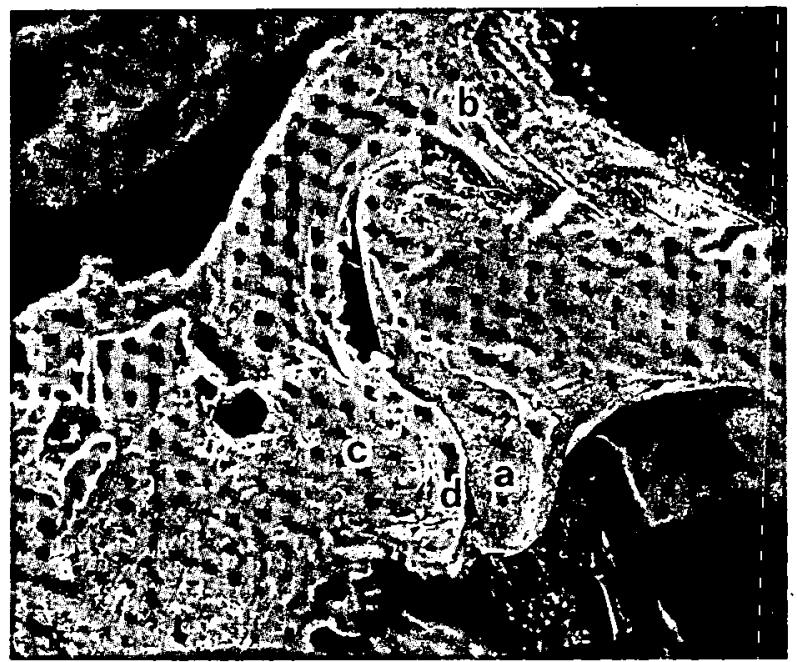

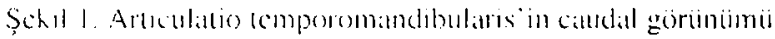
Finare 1. Caltadal view of the lemperomandibular joint. a. Citpul mandibula, b- m. masseler in pars profundals (profund

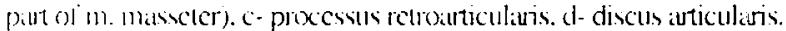

Anatomik terinlerde Nomina Analonica Velerinaria (19) esas almd.

\section{Bulgular}

\section{Articulatio temporomandibularis}

Articulatio temporomandibularis in occlusial (çiğneme) diizlemin yukarısinda yer aldığ belirlendi. I aleromedial yönde olan eklem yiizlerinden. calpul man dibulac'nin yatik "S" ve konveks (Sckil la). Cissil mandibularis'in ise konkav ve processus remaralicularis (Sckil lc) ile derin bir oluk şeklinde olduğu saptand. Articulatlo temporomandibularis'i örten eklem kapsulasinun ince ol duğu, caudal’inin ligamentum laterale (Şckil 21), diğcer bölümlerinin sse çiğneme kaslan ile destcklendiğ göruildii (Şekil 1b).

Discas articularis: Discus anticularis in (Şckil ld ve 4d) eklem kapsulasma bağ lanarak (Şckil $4 k$ ). cavum al

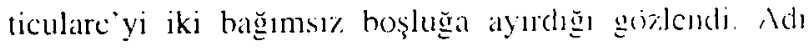
geçen oluşumun merkezinin ince. kenarlarmm ise (i/el likle rostral uçta belirgin bir kalınlık gösterdig̣ lespil catil di (Sekil 4). Discus articularis in medial'inde, dorsalle doğru ve fissura petrotympanica'ya kadau uzanan bir kal lunlaşma göruiddiu. Fibröz yapuda olan discus articularis in

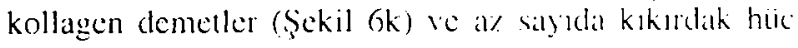
resinden (Şckil 5a) oluşuğ belirlendi. Bu demetlerm ya munda caudal uçta ve merkezde larkl yinnlerde seyreden elastik ipliklere (Şekil 5c) de seyrek olarak rastland. Discus articularis'in rostral ucunda yoğgun. merkezinde scy.. rek olarak kar damaları göriildii (Şckil ba).

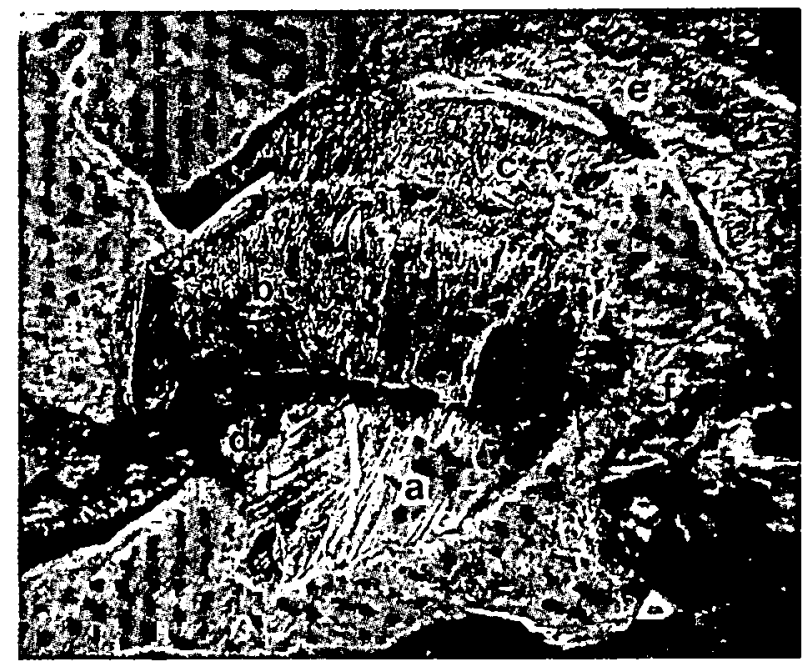

Şckil 2. Çiğncme kaslarmun lancral'den görtuntimui.

Figure 2. Appearance from latcrill of the masts attery muscles

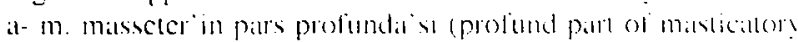

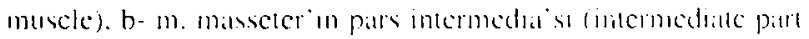

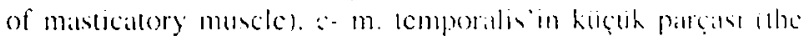

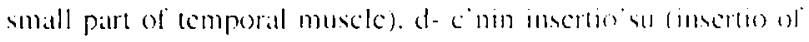

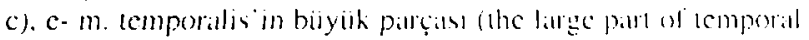
muscle), f- ligancutum latcralle. 


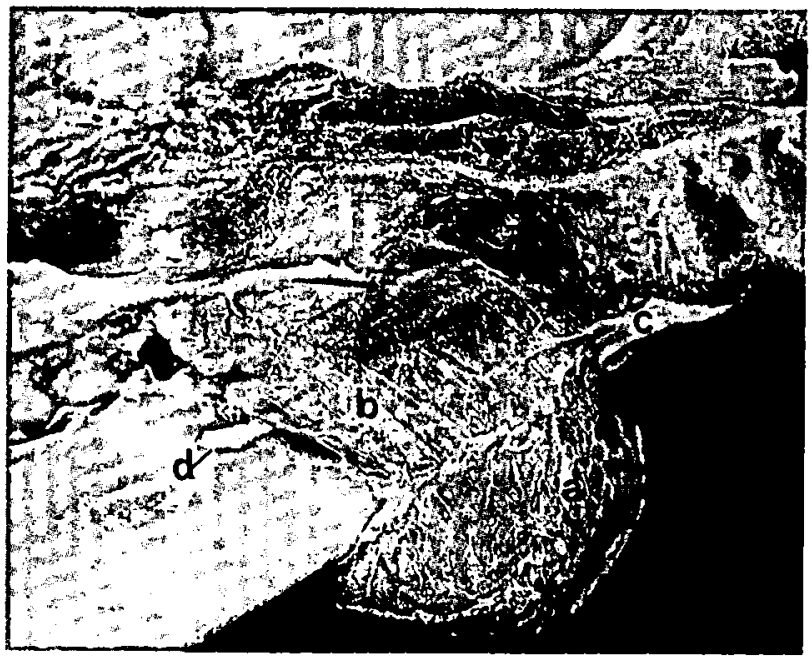

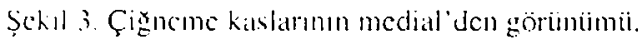

Figule 3. Appearince from medial of the masticatory muscles. i-117. manseler'in pars superficialis'i (superficial part of masticitory muscle), b- m. perygoideus medialis. c- a ve b hastartmen olusiurduge yapl (the form structured by a and b musctesio d- nervus lingualis.

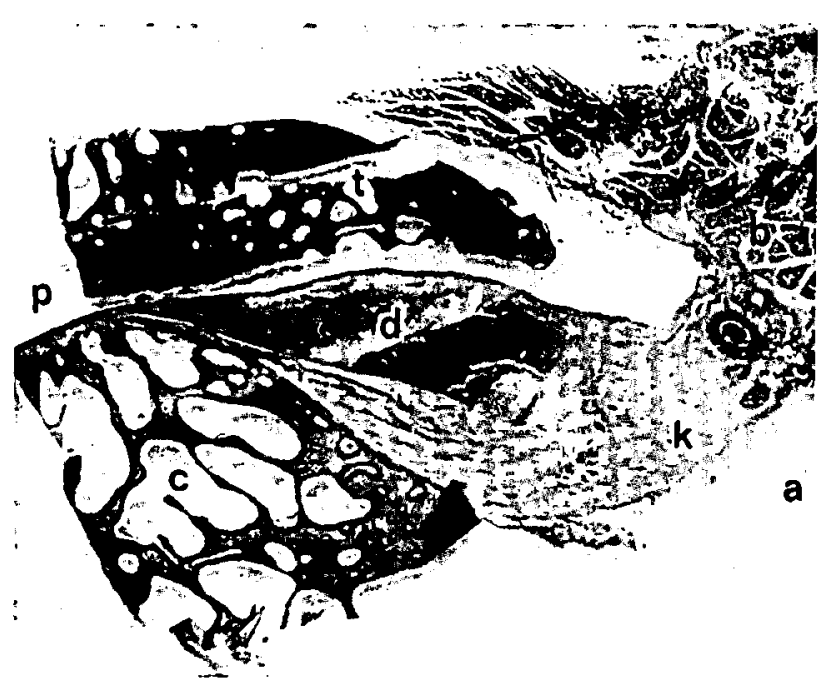

Schil 4. Articulatio temporomandibularis. Crossmon'un ïç̨ï hovilllitsi $\times 12$

ligure 4. Temporomandibulall juint. Crossmon's triple stain $x 12$. a- inlerior. b m. temporalis. c- caput mandihulace d- discus articularis. h-cipsula articularis. p-postcrior.

(iğneme kasları (Mm. masticatores)

Cigneme kistars. m. masseter, m. icmporalis, $m$ peryendeus medialis ve $\mathrm{m}$. perygoideus lateralis olarak incelendi.

Musculus masseter: Ü̧̨ bölümden meydana gelen hu kasın pars superlecialis'inin (Şekil 3a). juga alve(ularia dan ve os zygomaticum ile arcus zygonaticus'un lakral inden haşladığ ve kurvetli olduğu göribldui. Lifkrinin yönu caludo-ventral olarak belirlendi. Kasın çoğu lilinin ramus mandibulac nin ventral kenarma ve pro-

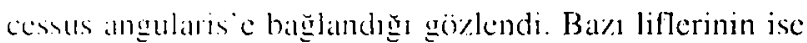

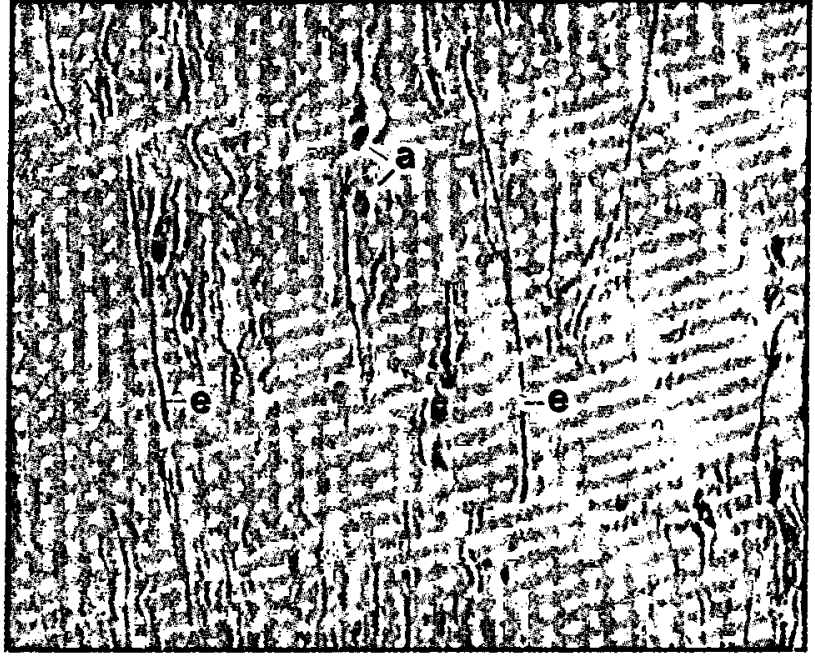

Sckil 5. Discus articularis de elastik ipliklerin sorimumu. Or ccin boyatna $\times 320$.

Figure 5. Appearance of elastic fibers in the anticulate disc. Orccins stain $\times 320$.

a- klkırdak hücresi (cartilalge cell). e- clastik iplikler (clastic libers).

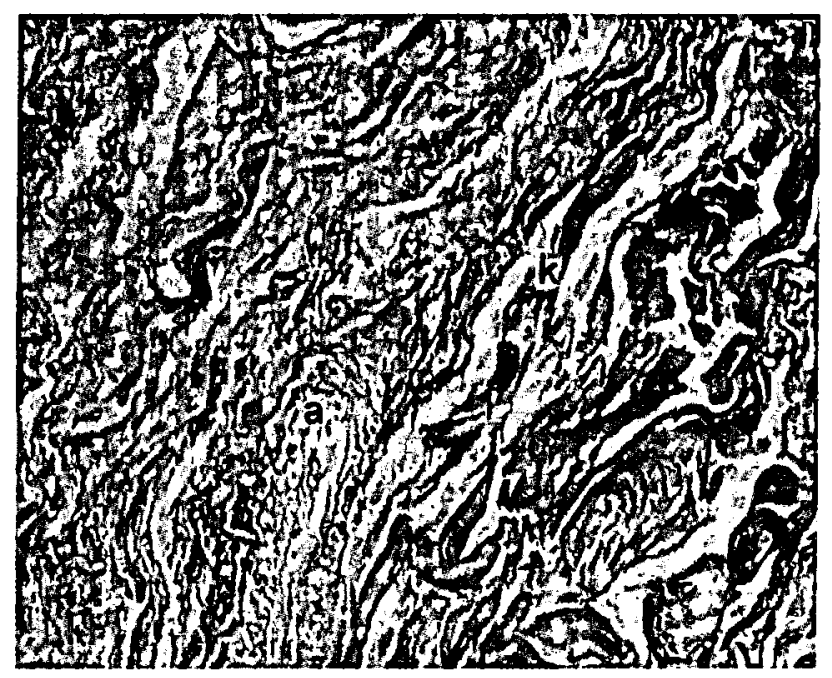

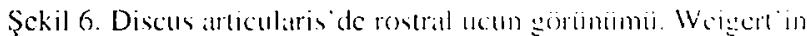
clastik boyamasi. $\times 30()$.

Figure 6. Appearance of rostral edece in the articulat disc. Blastac stain Weigert's $\times 360)$.

at-atter, $k$-kollagen demeller icollagen bunches).

m. pterygoideus mediališn lifleri (Şckil 3h) ilc hir leşerek (Sckil 3c) cartilatgo meallus acustici ile pom acusticus extertus"a kadar uzanan tendinim bir olușum scikillendirdiği lespil edildi (Sckil 3d). Pars incemedial an (Sckil 2b), arcis zygomaticus'un ventral kenalrmdan bas ladığı ve ventral olarak seyreden litherinin lossia masseterica ile ramus mandibulac nin arasma hagland tand. Pars profunda nun (Şckif 2a), arcus zy gomalicus"un medial yüzunden ve m. lemporalis in lilleri ile karrsmes olarak başladığ göruildiu. Bu böluimiun nervus massetericus ile iki parçaya ayndlığ rostral’inde yer alan 
patŗasmun crista coronoideus'a ve fossa masseterica nun dersal inc bağ landiğ ayruca rostro-ventral seyrettiği tespil edildi. Bu parçaumn derinde yer alan liflerinin horironlal olarak seyrentag ve processus coronoideus'a bağlandhğ göriildii. Caudal deki parçasının ise fossa massecurical j’ı doldurduğ ve rostro-ventral yönde seyretliğ belirlendi.

Mursulus temporalis: Fossa temporalis'den başlang̣ç alan hu kas iki parçi olarak incelendi. Küçiik olan patçasmun (S.Sekil 2c). arcus sygonaticus'un üstünde kavisli bir sekilde seyrederek, processus coronoideus un laloral yeizunc ve rostral ine hağlandığ görildii (Sekil 2d). Kalsın diğge parçasınun (Şckil 2e). ramus mandibulac ve processus coronoideus'un medial'ine bağlandığ beliflendi.

Musculus ptergogideus medialis: Bu kasm. os palatimum un lamina perpendicularis i. os presphenoidak. nin alast ve os pterygoidem un lateral yizü ile. hamulus plerygoideus ve os basisphenoidale' nin processus peryggoideus’undan başladığı görüldui (Şekil 3b). Ramus mandihulacinin ventral kenaranda ve processus angularis in medial yüründe sonlandiğ ve litlerinin caludoventral seyirli olduğ tespil cedildi.

Musculus pterygodens lateralis: Musculus pterygoideus lateralisin. foramen opticum ile fissura orhilališin alundaki çukurdan haşlang̨̧̧ aldığ belirlendi. lovea plerygoidea ya ve processus condylaris e bağlanan kism. liflerinin rostro-caudal yönde olduğu saptandı.

\section{Tartışma ve Sonuç}

Hayvansial kalynaklarla beslenen Alman çoban köpeğgnin articulatio) lomporomandibularis ve çiğneme kaslarmun antlomisi incelenmiştir.

Articulatio temporomandibularis literatürde (9) ifade cdildiğ gibi. occlusial (çiğneme) düzlemin yukarrsmala göriilmüş̧iur. Fox (11) kedide. Strön ve ark. (24) kopekle articulatio lemporomandibulais in çiğnene diirlemiyle aym seviyede olduğunu bildirmişlerdir. Ekkem kapsulass caudalonin ligamentum laterale ile 17.21).24). diğer kismlanmun ise fiğneme kaslan laralindan desteklendiğ ifade edilmiş $(3,10.15 .22,27)$, yaplan ب̣alışmakla da ayn bulgular elde edilmiştir.

Baßı araşuraciar (12.2()) discus articularis’in. laleral de capue mandibulae'ye. medial'de ise os temporalcije. diğgerleri (10.18) isc eklem kapsulasına bağlanhğgn bildirmişlerdir. Yaprlan çalışada discus atlicularis”in cklem kapsulasma bağlandığ görülmü̧̧ür.

Bu çalışnada. literatuir $(12,13)$ bilgilerine uygun bluak diskin lïbroz yapuda olduğgnu belirlenmiştir. Elas- tik ipliklerin diskin merke»inde de göriilmesi ve ösllikle diskin rostral’inde fazla damarlaşnamm olmass li-

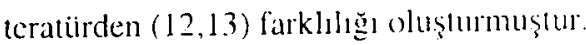

Besinlerin çiğnennesinde yal dar mandibutal ha reketlerin meydana gelnesinde etkin role sihup olan fagneme kasları litcraturde $(22,24.25 .27)$ tammlandığ gihj m. masseter, m. kemporalis, m. perygoideus lateralis we m. pterygoideus medialis olarak incelenmiștir.

Araşturmada kullanılan hayvanlarda m. masscler: pars superficialis. pars incermedia ve pars profunda olmak ä\%ere iiç parça halinde incelenmiștir. Pars superfictalus in

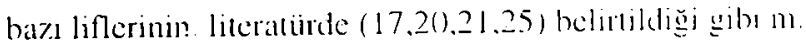
pterygoideus medialis ile birleştiği ve Scapino (2l) nun. meatoraphel ligament olarak isimlendirdigi ligamenti oluşturmuştur. Musculus masscter’ in orta parçası ve pars profundasi literature (18) benzer yapudader. Pars profunda rostral ve caludal olmak ï\%ere iki kısma ay rulmestur. Rostral bölüm Scapino (20). Tomo ve ark. (25) taralindinn m. zygomaticomandibularis olarak ifade colilmistir. Yapulan çalışmada m. masseter'on ụ̧̈ yaprağma ail lillerinin yönleri literaturle $(20,24)$ uyumlu de liflerinin yönui kala düzlemine göre lanmlanmmşır. Strom ve ark. (24) ve Scapino (20)'nun ise kas lillerinin yinuinu viicut düzlenine göre ifade etliklerj an laşılnakiadır.

Litcratürde (17.20.24) bittiun bir kas olatrak iliade celi ken m. tenıporalis, yapslan f̧alışmala iki bölim olatrak incelenniştir. Tomo ve ark. (25) aym kiss liç kımal ayırmış. m. cemporalis superlicialis olarak ifade elliklen kasın. yap̧lan çalışmada m. temporalis’in kiiçuik parçaısı olduğ belirlenmiştir.

Bu çalışnada m. pterygoideus medialis le m. plery-

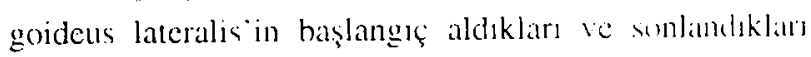
yerlerin literatiir (17.20.24-26) bilgilerinc useun ohlugu belirlenmiş ve add geçen kaslar hir hüllin olaralk incelenmiştir. Musculus plerygoideus lalcralis masmda

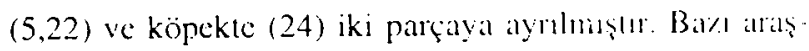
tıricılar $(9.20,26)$ köpekle bu kass hiiluin olaralk in.

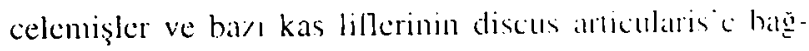
landığ incelenen m. plerygoideus latcralis in discus articularts bağlanmadığ̣ görülmüiştiir.

Bahsedien fiğneme kaslan dıșmelit. dumuzdal (23) ve köpektc (20.25) m. zygematicomilndibulatis ad re. rilen hir kas bildirilmiştir. Yaplan f̧allsosmada adı goęęn kas Ström ve ark. (24)’nun if adelcrinc hem\%er sekilele m. masscter'in derin yaprağ (pars profunda) olatrak incelenniştir.

Alman f̧oban koppeğande articulatio lempenomandi bularis ve çiğneme kaslanmun anaımmist incelemmiştir. Fik 
Lemi sckillendiren capu mandibulae' nin çok belirgin olan diș biikey yapısı ve processus retroarticularis’in çok gelişmiş olması, ayrıca discus articularis in rostral ucunun helirg̣in kahmlığ rekellerini daha fazla yapabildiğini gösterir. Discus arlicularis "in fubröz yapıda olması ve elastik lifler içermesi, eklem iizerine düşen kuvvellere karşı diskin dayanıklılıפ̆ kuvetli bir yapida olan ligamentum laterale ile desıeklemmiş olması. eklem içi uyğulamaları zorlaşırır. Musculus masseler; pars superficialis, pars intermedia ve pars profunda olarak incelenmiştir. Musculus temporalis iki parça halindedir. Bu iki kasın iyi gelişmiş olmaları. rolasyon harcketinin daha güçlii yapıldığını göstcrmektcdir.

\section{Kaynaklar}

1. Açıkalın E, Ünal N, Ortuğ G (199)()): Cene eklem kapsialiumiun morfolojik re fonksivonel incelenmesi. Anadolu Tip Derg. 13. 15.22

2. Arıne K, Wlhan A (1995): Anatomi. Kemikler. Eklemler. Kàslar ve lç organlar. 1. Cilt. Giunes Kitap Evi Led Şti. Ankillial.

3. Bade H. Schenck C. Kocbke J (1994): The funcrion of discomussular relationships in the human temporomandiInelar foim Acta Ant1. 151. 258-267.

4. Bermejo A, Gonzalez O, (jonzalez JM (1993): The pig as an anumal model for experimenation on the tempremendithalar articular amplex. Oral Surg Oral Med Oral Pallhol. 75, 18-23.

5. Carpentier P. Yung JP, Bonnet RM, Meunissier $M$ (1988): Insertions of the lateral plengoid muscle: An anaamic suad of the heman temporomandibular joint. J Oral Maxillolia Surgy. 46. 477-482.

() Crossmon (; 1937): A modification of Mallory's connective lissue stain with a discussion of the principles inwhe. Anall Rac. 69. 33-38

7. Culling CRA, Allison R'I, Barr WT (1985): Cellular Palholes... Technugue. Fourth Ed. Butlorworths. I ondon.

8. Demirsoy A (1992): Yasamun Temel Kurallart, Omur-

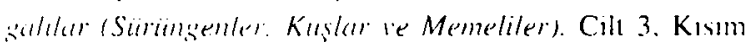
2. Bilium 42. Birinci Baski. Metcksin AS. Ankarit.

1) Dyce KM, Sack WO, Wensing CJG (1987): Texthosk of the Verterinaly Anatumy: WW Saunders Company. Philiklciphia

10. Fenoll AB, Sequeros OG, Gonzalez .JMG (1992): His-

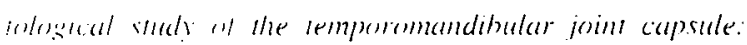
Therey of the ariculke comples Actat Anat, 145. 24-28.

il Fox SS (19)51. Lateral haw menement in mammalian dentiltsils. I Prosth Dent. 15.81()-825.
12. Gillbe GV (1973): A comparison of the dise in the crot niomandihular joint of the mammals. Actal Anall 86. 394. 409.

13. Gillbe GV (1975): The function of the dise of the lemporronandibular joimt. J Prosth Dent. 86. 394-4(14).

14. Luna I.G (1968): Mamual of Histrilogite: Sicumings Methods of the Armed forres Instiute of Patholowe. Thind bal. McGraw-Hill Book Compiny, New York.

15. McKay (iS, Yemm R. Cadden SW (1992): The structur,

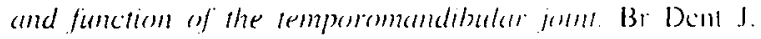
173. $127-132$

16. Meister VR, Berg $\mathbf{R}(1974)$ : Untersuchungen wher die form. Grobe und dicke der disci arficulares articalatums temporomandibularis wom rind. sehal. schwein and huend. Anal An7. 135. 24-34.

17. Miller ME, Christensen GC, Evans HE (1965): Anctum! of the Dog. WB Situnders Company. Philindelphiat.

18. Nickel $\mathbf{R}$, Schummer $\mathbf{A}$, Seiferle $\mathbf{H}(1986)$ : The Anculmm of the Domestic Animals. Vol I. Verline Palul Pillcy. Bcrlin.

19. Nomina Anatomica Veterinaria (1994) Puhlivhed by the

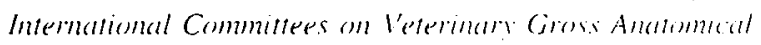
Nomenckuture. Fourth Ed. New York.

20. Scapino RP (1965): The third joint of the cominte jont: I Morph, 116. 23-50.

21. Scapino RP (1974): Function of the masseter-pleregend raphe in camivores. Anal Ans. 136, 4311-446.

22. Schmolke C (1994): The relationship between the tem-

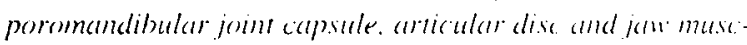
les. J Anal. 184. 335-34.5

23. Strön D, Holm S, Clemensson E. Haraldson T (1986) Gross anatrmy of the mandibular jomb and masticatrin muscles in the ammestic pig ISus scrotaj. Arch Oril Biol. 31. 763-768.

24. Ström D, Holm S, Clencensson F, Haraldson T (1988)

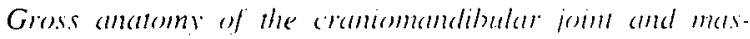
ticatory muscles of the dese. Arch Orat Biol. 33. 597-6014.

25. Tomo S, Hirakawa T, Nakajina K. Tomo I, Kobayashi S (1993): Morpholegical claseffication of the mersucarom muscles in dogs based on their innervarion. Ann Allill. 175. 373-380.

26. Tomo S, Hirakawa T, Nakajima K, Tomo I, Kobayashi S (1995): The monphotosy and innervation of the lateral

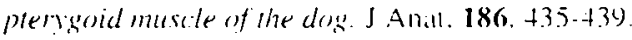

27. Velasco JRM. Varquez JFR, Collado J.I (199)3): The te-

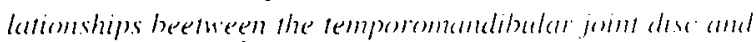
related masticanorid mascles an humans. I Orall Maxillolia Surg. 51, 39) - 395 .

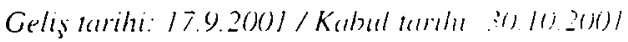

Yazışnıı adresi:

Yrd.Dog. I)r.Maral Kallok

Mussata Kemal L'mlensilesi

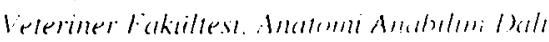

Anakiar, Hatar

e-mail: muratkubaks!mku.edu.ts 